\title{
An Ontology-Based Framework for Modeling User Behavior A Case Study in Knowledge Management
}

\author{
Razmerita, Liana
}

Document Version

Final published version

Published in:

I E E E Transactions on Systems, Man and Cybernetics, Part A: Systems \& Humans

Publication date:

2011

\section{License \\ CC BY-NC-ND}

Citation for published version (APA):

Razmerita, L. (2011). An Ontology-Based Framework for Modeling User Behavior: A Case Study in Knowledge Management. I E E E Transactions on Systems, Man and Cybernetics, Part A: Systems \& Humans, 41(4), 772783.

Link to publication in CBS Research Portal

\section{General rights}

Copyright and moral rights for the publications made accessible in the public portal are retained by the authors and/or other copyright owners and it is a condition of accessing publications that users recognise and abide by the legal requirements associated with these rights.

Take down policy

If you believe that this document breaches copyright please contact us (research.lib@cbs.dk) providing details, and we will remove access to the work immediately and investigate your claim. 


\title{
An Ontology-based Framework for Modeling User Behavior - A Case Study in Knowledge Management
}

\author{
Liana Razmerita
}

\begin{abstract}
This article focuses on the role of user modeling and semantic-enhanced representations for personalization. The paper presents a generic Ontology-based User Modeling framework (OntobUMf), its components and its associated user modeling processes. This framework models the behavior of the users and classifies its users according to their behavior. The user ontology is the backbone of OntobUMf and has been designed according to the Information Management System Learning Information Package (IMS LIP). The user ontology includes a behavior concept that extends IMS LIP specification and defines characteristics of the users interacting with the system. Concrete examples of how OntobUMf is used in the context of a Knowledge Management System (KMS) are provided. The paper discusses some of the implications of ontology-based user modeling for semantic-enhanced Knowledge Management (KM), and in particular for personal KM. The results of this research may contribute to the development of other frameworks for modeling user behavior, other semantic-enhanced user modeling frameworks or other semantic-enhanced information systems.
\end{abstract}

Index Terms - personalization, user modeling, knowledge sharing, semantic-enhanced knowledge management, semantic web, ontology, web services

\section{Towards Personal KnOwledge Management}

As knowledge is at the center of most of human activities, managing knowledge and Knowledge Management (KM) are an important endeavor for individuals and organizations. Even though there are many non-technological facets of KM research and practice, modern knowledge management is inseparable from a consideration of technology [1] and emerging technologies such as social/semantic web. Knowledge Management Systems (KMS) are information systems dedicated to manage organizational knowledge [2]. A

Manuscript received November 16, 2008; revised in June, 2009, July 2010 and Accepted September 20, 2010.

Liana Razmerita is Assistant Professor at Copenhagen Business School, Denmark. (liana.razmerita@cbs.dk).

Part of the work reported in this paper was done in the context of the Ontologging project, an EU funded project. Ontologging system has been developed by the Ontologging consortium: CALT (Center of Advanced Learning Technologies), INSEAD, France, INDRA and Meta4, Spain, Archetypon, Greece, FZI, Germany and Deltatec, Belgium. Special thanks are due to Albert Angehrn, Thierry Nabeth and to the anonymous reviewers of this article. first generation of KMS aims to enable simple ways of storing, accessing, sharing and using knowledge more effectively. Traditionally corporate KMS consist of databases, intranets and groupware systems [3]. Formal processes associated with the management of knowledge are timeconsuming, perceived as cumbersome to use and often have represented a barrier for the use of the systems. Modern KM tools will enable easy, free authoring and content creation (through blogs, wikis) and in addition better structure knowledge and better support knowledge work (e.g. finding and retrieving information). The focus of new knowledge management systems and practices is placed on sharing and creating knowledge which in turn leads to innovation and improved effectiveness at both individual and corporate levels [4].

The emergence of Social and Semantic Web technologies has brought new possibilities to interact, communicate, share knowledge, collaborate and manage knowledge. New generations of KMS go beyond the mere administration of data; they will better support the management of tacit knowledge, learning processes, knowledge creation, knowledge sharing and collaboration between employees, irrespective of their location, using a variety of new applications such as: social networks or semantic social networks, wikis or semantic wikis, blogging or semantic blogging and/or micro-blogging. New KM tools exploit the new opportunities of sharing knowledge and interacting with customers, suppliers and partners taking advantage of new social, collaborative services and tools available on the web and in distributed computer networks. Furthermore, these new emerging killer applications combine sharing information, knowledge and social dimension and undermine principles like: information asymmetry and top-down content delivery [5]. Semantic Web technology is key for moving towards collaborative, semantic-based KM [6]. In the context of web access, only authorized individuals must be permitted to execute various operations and functions in an organization and secure knowledge management must be enforced. Secure knowledge management is critical as organizations have to protect their intellectual assets [7]. More recently, the concept of personal knowledge management has been developed. While the traditional view of $\mathrm{KM}$ is placed on the management of organizational knowledge, the collective knowledge, the personal $\mathrm{KM}$ is focused on the individual on his quest to find, learn, share, knowledge and socialize [8]. 
One of the key objectives of personal KM is to help individuals become more effective, supporting his/ her rapid development, learning new skills and knowledge [9].

In this paper, it is argued that user modeling processes and personalization mechanisms can contribute to bridge the gap between KM and personal KM. Personalization of a KMS is the process that enables interface customization, adaptations of the functionality, structure, content and modality in order to increase its relevance for its individual users [10]. Personalization is a pervasive phenomenon in all human activity, encompassing decoration reconfiguration, modification, customization of software or human-made objects [11]. Within this work personalization is extended beyond customization and adaptation mechanisms at the level of the user's interface; the emphasis is placed on personalized user support for knowledge workers. This article places special emphasis on the link between user modeling and the support of knowledge worker's activities such as: create knowledge, share knowledge, learn and get feedback based on his/her activity in the system. This article focuses on the role of semantic-enhanced user modeling services for personalized user support within KMS. The paper presents a generic framework for modeling users' behavior based on ontologies (OntobUMf). The model of users' behavior includes three sub-behaviors, level of knowledge sharing, level of activity, type of activity. OntobUMf was designed and implemented in the context of the Ontologging project. Ontologging has developed methods and tools for corporate ontology formalization and formal ontology definition methods for Knowledge Management. Based on the analysis of the evaluation results, this article shows that adaptation methods and personalization techniques relate to specific needs of the knowledge workers and specific challenges and objectives of KM systems. Knowledge workers are autonomous and cannot be coerced to share or provide knowledge. They must be motivated and/or they should perceive the benefits of using such a system. Knowledge workers want systems that enable to access timely the "right" information, reuse past experience, be able locate experts in the organization and save time. At an individual level, these specific needs, relate to a personal dimension of KM, while at an organizational level, general objectives and challenges of managing knowledge relate to a more collective dimension of KM. Among these general objectives and challenges are: How to motivate knowledge workers to share knowledge? How to facilitate/stimulate collaboration between knowledge workers irrespective of their location? How to simplify business processes and work tasks? How to alleviate information overload? How to foster innovation and creation of new knowledge?

The remaining part of the article is structured as follows. The second part includes a literature review of the ontologybased user modeling and semantic-enhanced KM. The third section describes the ontology-based user modeling framework (OntobUMf), its different components and the associated user modeling processes. The fourth section discusses some evaluation results, some of the lessons learned and its possible implications for future developments of semantic-enhanced KMS. The last part includes conclusions and future lines of research.

\section{LITERATURE REVIEW}

In the last few years, the concept of ontology has started to be used in connection with Semantic Web research. Ontologybased representations are powerful representation structures. The usage and application of ontologies are increasingly seen as the key to enable semantics-driven data access and processing [12] or semantic-enhanced search. An ontology can be defined as a set of knowledge terms, including the vocabulary, the semantic interconnections which can be associated with inferences, "inferencing" and smart queries for any particular domain. Inferences or "inferencing" means that given some stated information one can determine other related information that one can consider as it had been stated [13]. Ontology languages can be classified based on the knowledge representation formalism in which they are represented: enriched first order predicate languages, framebased approaches and description logics. Semantic Web research has devoted an important effort in defining a common language for ontology modeling and reasoning with the objective to achieve semantic interoperability. The Web Ontology Language (OWL), a language based on description logic has become the recommended language by the World Wide Consortium in 2004. However, adding additional layers and especially a rule layer on top of OWL is still a central task for the Semantic Web [14]. Rules and associated reasoning mechanisms are important for instilling intelligence on the Semantic Web, and in particular connecting and making better use of the data, information and the collective knowledge harnessed by the various types of applications. Rules and metadata along with user's preferences, goals, needs, and interests stored as a user ontology will constitute the core underlying architecture for Human Semantic Web. A human or user-centric approach will imply a change from the current "push approach" towards a personalized "pull approach" in knowledge and learning management paradigm [15]. User's interests, goals, needs could be a basis for achieving this pull approach and furthermore be a basis for establishing collaborations, networking with other peers, friends or colleagues. In the evolution of the web, three different semantic stages can be distinguished: semantic isolation, semantic coexistence and semantic collaboration [15]. These stages will eventually lead to semantic interoperability or "semantic collaboration" which are key for achieving the Human Semantic Web vision [15].

The development of Semantic Web technology has created the context of different semantic-enhanced user models either for the purpose of creating distributed user/learner models [16] or/and as an attempt to share them across different types of applications [17, 18]. Previous user or student models were represented by means of logic-based formalisms such as predicate logic and later using semantic networks, conceptual graphs, databases, frames and objects. The logic-based formalisms, such as predicate logic have the advantage of simple, well-defined associated reasoning mechanisms, but 
they lack structuring properties. Non logic-based formalisms are object-oriented representations, rule-based representations, neural networks, semantic networks, conceptual graphs, etc. Non-logical representation formalisms are more appealing, more intuitive and easier to understand by non-experts. In the past few years, ontology-based user modeling has been proposed for different application scenarios. User modeling associated rules and ontology-based representations for realtime ubiquitous applications in an interactive museum scenario have been proposed by [19]. In a ubiquitous computing scenario users can delegate tasks to different agents acting on various devices with computational capability. Context features and situational statements for ubiquitous computing have been proposed as a General User Model Ontology (GUMO) by [17, 18]. The use of semanticenhanced user profiles for web search applications has been proposed in $[20,21]$. Another strand of research emphasizes the use of ontology for adapted learning content, semantic learning portals [22] that constitute dynamic smart learning spaces [22-25]. In the context of the semantic web the specification of standards for the management of personal identities has a great potential for providing intelligent learning services [26]. Semantic web and ontologies may be a catalyst for learning organizations where ontology-based competency management plays a central role [27].

Up to now, little detailed work has been done related to ontology-based or semantic-enhanced user modeling in relation with modeling the user behavior for managing knowledge. The user model proposed in FRODO project focuses on the user tasks and role which are associated with specific information needs. The tasks at hand are triggering different information needs. Furthermore different persons may have varying information needs with respect to the same tasks, depending on their personal skills and knowledge [28]. The user ontology described in the On-To-Knowledge project [29] uses manually constructed ontologies about skills, job functions, and education. This ontology is dedicated for skills management application.

In semantic-enhanced information systems, the ontology represents and structures the different knowledge sources in its business domain aiming to improve the overall performance of the system [25, 30-33]. Existing knowledge sources (documents, reports, videos, etc.) are mapped into the domain ontology and are semantically enriched. This semantically-enriched information enables better knowledge indexing and searching processes and implicitly a better management of knowledge. Ontologies offer a flexible and expressive layer of abstraction, very useful for capturing the information of repositories and facilitating their retrieval either by the user or by the system to support the user tasks [34]. An ontology-based system can be used not only to improve precision of search/retrieval mechanism but also to reduce search time [35]. For these reasons, ontology-based approaches will likely be the core technology for the development of a next generation of semantic-enhanced KM solutions. However bringing semantic-enhanced information systems to a real world enterprise application is still a challenge. One reason could be that ontology-based conceptual representations lack certain features which are important for classical database driven information systems. Features such as scalability, persistence, reliability, and transactions standardized in classical data-base driven applications are typically not available in ontology-based systems [36]. Furthermore an ontology-based semantic markup can be used as a machine interpretable format for software agents and semantic web services to operate. Annotations with well defined semantics (metadata) can be provided using semantic annotation tools. Authoring annotations for legacy resources is an important effort and advanced semantic annotation tools need to be made available in order to provide effective semantic-enhanced KM systems [37].

\section{ONTOLOGY-BASED USER MODELING FRAMEWORK (ONTOBUMF)}

OntobUMf has been designed as a three-tiered application server dedicated to manage information about users [38, 39]. The server is generic, designed as a modular architecture that may be extended and used for any application domain. The user modeling server acquires and maintains the user's data through a user profile editor (explicitly) and through different user modeling techniques (implicitly). As represented in Figure 1, the layers consist of: 1) the user front end layer on top, 2) a middleware layer or service layer, and 3) an ontology and a data layer at the bottom.

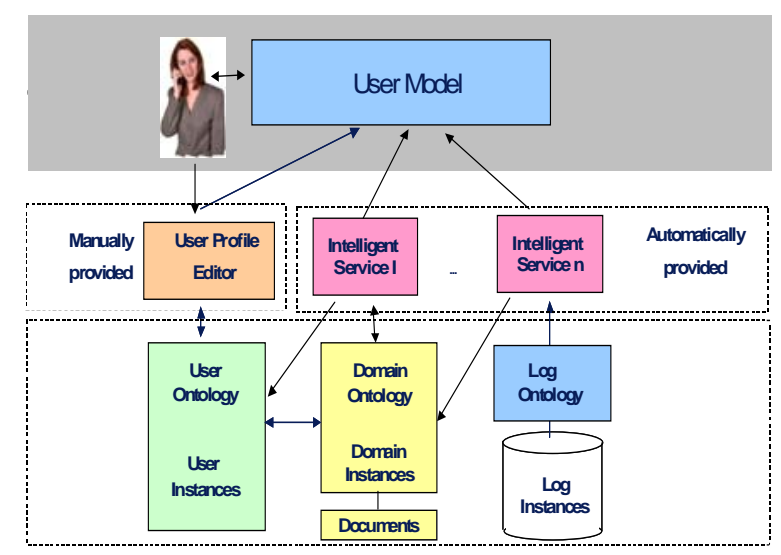

Figure 1: Ontology-based user modeling architecture

The user front end layer consists of tools that enable the user to access and update his/her user model or user profile. The user profile editor is specialized ontology editors dedicated to instantiate and/or visualize the user ontology. The user profile (see Figure 2) initializes the user model but it also enables users to visualize it, and update it. Furthermore this tool enables users to scrutinize their behavior, their level of activity, the type of activity and the level of knowledge sharing. The user model is an open user model which is expected to create awareness of the identified behavioral model, enable comparison with other users, provide feedback 
and maybe activate a social norm of behaviors. Recent research suggests that with the appropriate interface, a user ontology may become an easily customizable repository of information that may serve as a memory aide for the user and a tool that may be used for personal information management [34].

The service layer includes several dedicated intelligent services. The user modeling techniques and the personalization mechanisms are represented as intelligent services. Service layer is responsible for handling requests and communicating with the data layer. Services can access the user ontology which is persistent on the server. The user model can be accessed directly by invoking functions of a web service in order to provide personalized/intelligent services. OntobUMf has a modular architecture which allows adding incrementally different intelligent services. The layering associated with the modular design allows bundling the functionalities provided. The services have two main roles in the system:

- to update and maintain the user model on the basis of usage data available from the running system through the category extractor. The category extractor integrates specific mechanisms for modeling the characteristics of the users interacting with a KMS. Other types of inferences and reasoning mechanisms specific to other application domains can be defined and integrated in the future.

- to provide a set of personalized services based on the characteristics of the users. A personalized service tailor the information provided taking into account the user's characteristics (e.g. interests, type of activity, expertise or context). The personalization of a KMS may be defined as the process that enables interface customization, adaptations of the functionality, structure, content and modality in order to increase its relevance for its individual users [10]. The adaptation techniques, can be classified into three categories: adaptation of structure, adaptation of content, adaptation of modality and presentation [40]. Personalized services include a multi agent-system that extracts information from the user profiles. The multi-agent based architecture include: a user agent, a yellow page agent, an interest monitor, a secretary agent and a number of conflict-detection agents. A further description of the multi agent-system can be found in [41]. This multi-agent system focuses on coordination and cooperation agents. Security considerations and security policies have also to be considered for sharing knowledge in virtual communities for distributed knowledge management by means of normative multi-agent systems [42]. Agents can be knowledge providers who respect global policies. The rules of policies for secure knowledge management do not concern only what knowledge the users are prohibited or permitted to access, but they also concern which regulations the knowledge providers are allowed or obliged to enforce [42].

The data layer includes user data which is related to domain specific data. The semantics associated with the domain and user model is mapped into the user and domain ontology. Ontologies capture the concepts and the relationships between the concepts describing the different resources available in the system. OntobUMf has access to three different ontologies. User's ontology concepts are related to the domain ontology concepts. The activity of the users in the system is described in the log ontology and it is captured as log instances. The ontologies are known and mapped manually at design time. Although semantic technologies are designed with extensibility and openness in mind, currently programming languages and tools are not able to fully exploit it. It is expected that future semantic applications will be using multiple ontologies, discover them and integrate them on request [43]

The user ontology structures the characteristics of the users in concepts, sub-concepts, properties and their relationships. The user ontology has been developed based on a top-down approach starting from IMS LIP specification, employing Ushold and Gruninger methodology [44]. It has been specified taking into consideration end-user requirements provided by two Spanish companies involved in the Ontologging ${ }^{1}$ project combined with research drawn from research fields such as: user modeling, adaptive hypermedia and user-adaptive interaction and knowledge management. The user ontology is conceptualized according to IMS LIP specification: "The intent of the specification is to define a set of packages that can be used to import data into or extract data from an IMS compliant learner information server." [45]

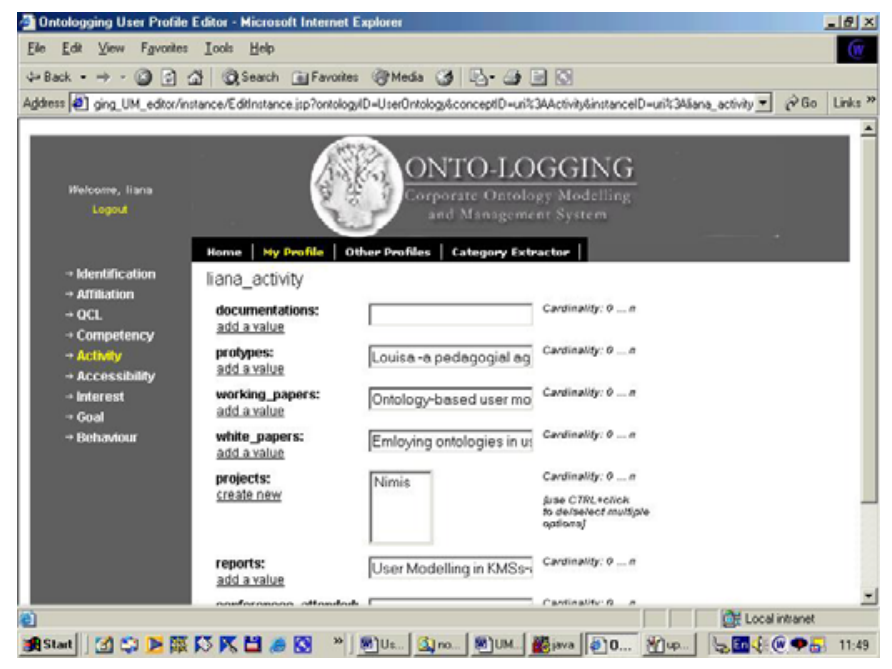

Figure 2 User Profile Editor, in the edit mode

IMS LIP package is structured in eleven groupings in order to enable learners to customize their experience and formulate it in a general form. These groupings include the following assimilated concepts: Identification, Goal, QCL (Qualifications, Certifications and Licenses), Accessibility, Activity, Competency, Interest, Affiliation, Security Key and Relationship. According to the IMS LIP specifications the

\footnotetext{
${ }^{1}$ http://www.ontologging.com/
} 
learner information can be packaged from a variety of systems that are not limited to human resource management, student information and/or learning management systems. A more detailed description of the way in which the ontology has been built can be found in [46].

Behavior is defined as a concept that models characteristics of a user interacting with a system. The Behavior concept is an extension of the existing IMS LIP concepts. Inferred fields grouped as Behavior are calculated based on the data extracted from the log files (the traces of the users). For a KM system heuristics and fuzzy logic rules allows the measurement of the Type_of_Activity, the Level_of_Activity and the Level_of_KnowledgeSharing of the users in a KMS. Apart from the Behavior concept which is specific for KMS, the user ontology is based on IMS LIP specifications. Therefore it is, to a large extent, domain independent and it can be applied or it can be extended to other application domains if necessary (e.g. Human Resources Management Systems, e-Learning, etc.). Some of the user's characteristics could be filled-in by the users using a user profile editor or imported in the user ontology while others can be inferred based on the user's interaction with the system. Some of the user's dimensions are static while others are dynamic; some features change fast while others can change slowly in time.

The domain ontology describes the domain knowledge in terms of concepts and relationships between various concepts. The domain ontology can conceptualize different application domains (e.g. e-commerce, e-Learning, etc.). The Ontologging domain ontology is centered around the administration of tenders. The domain ontology has been developed at different stages as the first domain ontology faced a series of problems at the usage stage. The terminology used in the everyday tasks by the knowledge workers was not the same in the conceptualization of the domain ontology. A set of too generic concepts were confusing for the end-users so they were misused or used for everything. Consequently, concepts which were too specific have never been used. A set of missing concepts has also been identified for usage. The ontology reengineering process of Ontologging project has emphasized the fact that achieving a shared conceptualization is not a straightforward process.

The log ontology defines the semantics of the user interaction with the system. It describes the user's actions and the associated events triggered in the system. These events, described through the $\log$ ontology, are captured as $\log$ instances. The specification and implementation of the ontology is generic enough to allow the ontology to be adapted to different application domains.

\subsection{Modeling User Behavior}

Modeling user behavior is an ongoing challenge in different application domains including Knowledge Management, ecommerce, decision support, e-learning, marketing. Such advanced systems allow having a better knowledge of the user (in terms of needs, preferences or goals) in order to offer him/her enhanced services and better support users. There are many dimensions and elements that need to be considered when building a user model for a specific application domain
[47]. Most of the studies of internet user behavior are based on statistics of user traces and traffic measures [48]. In this case, user behavior characterization takes into account statistics of user session duration, data rates, application popularity, user mobility in order to predict trends or make recommendations to the users. Researchers often make use of theories from sociology or psychology in an attempt to reveal the hidden rationality of the user browsing behavior [49], to understand individual differences and cognitive styles [50] or web browsing strategies and goals [51]. From a social perspective, user behavior has been studied in online communities in relation with behavioral patterns of collaboration in order to understand different forms of motivation [52], to evaluate knowledge sharing dilemma in order to design interventions to successfully manage organizational knowledge [53], or to understand the different categories of users and their motivation for participation in online communities [53].

The proposed model of user behavior, and in particular, the definition of the level of knowledge sharing concept makes use of the diffusion of innovation theoretical model [54]. Diffusion of innovation theory presents innovation as being communicated through certain channels over time, and within a particular social system. Individuals have different degrees of willingness to adopt innovations and thus it is generally observed that the portion of the population adopting an innovation is approximately normally distributed over time [54]. Breaking this normal distribution into segments leads to the segregation of individuals into five categories of individual in relation with their attitude towards adoption of innovation (from the earliest to the latest adopters): innovators, early adopters, early majority, late majority, laggards [54]. Members of each category typically possess certain distinguishing characteristics as briefly described below: innovators are venturesome, educated and use multiple sources of information; early adopters are social leaders, popular and educated; early majority think and make decisions carefully and use many informal social contacts; late majority are skeptical, traditional and usually have a lower socio-economic status; laggards use neighbors and friends as main source of information, they are characterized by fear of debt.

So, in order to define our model with respect to the level of knowledge sharing these five categories of users are mapped using Near's terminology into the following categories of behaviors: unaware, aware, interested, trial and adopter. Similar to the artificial intelligence models of behaviors, the behavior of the users is correlated with goals, beliefs, commitments reflected in the user's activity or attitudes. The attitude is defined as a predisposition to respond consistently with respect to a certain modeled task.

$\mathrm{B}=\left\{\mathrm{b}_{1}, \mathrm{~b}_{2}, \ldots, \mathrm{b}_{\mathrm{n}}\right\}$

B - a set of behaviors, in our case B consists of level of knowledge sharing, level of activity, type of activity

$\mathrm{A}=\left\{\mathrm{a}_{1}, \mathrm{a}_{2}, \ldots, \mathrm{a}_{\mathrm{m}}\right\}$

A- set of attitudes, in our case study the set of attitudes are consisting of events reflected in the level of activity, type of activity of the users. 
The exhibited user's behavior is modeled through a set of rules:

$$
\mathrm{r}:\left(b_{\mathrm{i}}->\mathrm{a}_{\mathrm{j}}, \alpha_{\mathrm{ij}}\right)
$$

$\alpha_{\mathrm{ij}}$ reliability degree of the rule

Such rules depict the approximate degree of a user behavior under the overall assessment of the different user's attitudes (sub-behaviors) which correspond to a membership degree in a fuzzy set. Furthermore these rules can be treated as a set of fuzzy sets on A, where the membership degree in a fuzzy set $f_{j}$, corresponding to an attitude $a_{j}$, is $\alpha_{i j}$.

$F_{j}$ is a set of rules for modeling user's behavior $b_{i}->a_{j}, \alpha_{i j}$ The Behavior concept and its sub-concepts (see Figure 3) were introduced to model two processes that are important for the effectiveness of a KMS, namely knowledge sharing and knowledge creation. Based on their activity in the system, namely the number of contributions to the system and the number of the documents, the user modeling system classifies the users into three categories: readers, writers or lurkers. These categories are properties of the type of activity concept. Readers are categories of users mostly accessing the resources of the system, while writers are accessing and contributing with resources, metadata to the system. A lurker is defined as somebody who doesn't contribute and who accesses very few knowledge assets in the system. Whenever a user contributes to the system an associated variable $\mathrm{nb}$ of contributions (NC) is incremented. Similar rules are triggered when the user opens, reads, queries, provide metadata (tag) or comments on resources available in a KM system.

$\forall \mathrm{xProvideResource-}>$ increase(nb_of_contributions $(\mathrm{x})$ )

$\forall \mathrm{x}$ ReadResource -> increase (nb_of_read_resouce(x))

$\forall \mathrm{x}$ ProvideMetadata $->$ increase(nb_of_read_resource(x))

$\forall x$ Query -> increase(nb_of_query(x))

The classification of users according to the type_of_activity or to the level of activity is based on heuristics taking into account the number of contributions (NC) and the number of accessed/read resources (NR).

If (nb_of_read_papers $>\mathrm{NR}$ ) and (nb_of_contributions $<\mathrm{NC}$ )

Then user $(\mathrm{x})=$ "reader" (during timeframe)

Where, NR, NC and timeframe are constants that can be parameterized depending on the activity in the system.

The level of activity defines four categories of users: very active, active, visitor or inactive. A very active user reads/accesses and contributes with knowledge assets. An active user has less activity in the system than a very active user. A visitor is somebody who rarely uses the system while the person classified as inactive has no activity in the system. An example of inference rule for classifying the users based on their level of activity is:

If (nb_of_read_papers $>$ NR) and (nb_of_contributions $>=\mathrm{NC}+1)$ Then user( $\mathrm{x})=$ "very active"(during timeframe)

OntobUMf uses the principle of fuzzy classifier systems to assign the users to a certain category according to their level of knowledge sharing. The user's states, in relation to the level of knowledge sharing, are: unaware, aware, interested, trial and adopter. Fuzzy logic is often used to model various types of common sense reasoning similar to a more humane way of thinking and reasoning. Fuzzy logic extends conventional
Boolean logic to handle ambiguity and uncertainty or partial truth. The value between completely true and false are determined by the membership function which takes value in the $[0,1]$. Fuzzy reasoning was introduced by Zadeh in 1960's to handle the uncertainty of natural language. Fuzzy logic research concentrates on approximate reasoning and reasoning under uncertainty issues. "Fuzzy logic aimed at a formalization of models of reasoning that are approximate rather than exact" [48]. It has been applied to various application domains like: knowledge-based systems, knowledge acquisition, control systems etc.

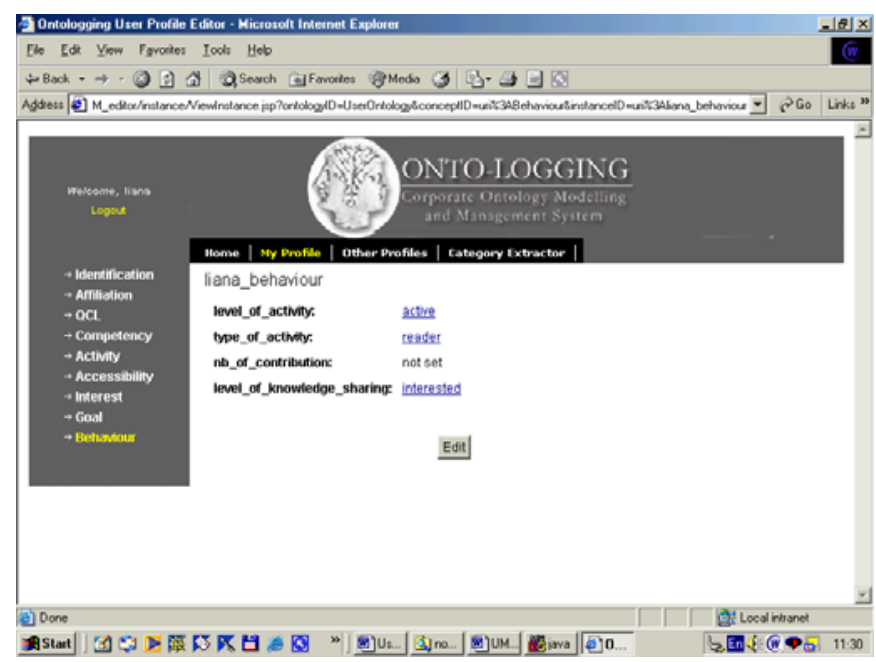

Figure 3 User Profile - the Behavior concept

We use the principle of fuzzy classifier systems in order to assign the users in different categories according to their level of knowledge sharing.

Fuzzy classifier systems imply a two-step process:

- to create a fine-grained fuzzy partition;

- to generate fuzzy rules and calculate membership function or degree of membership;

Through the level_of_knowledge_sharing, OntobUmf captures the level of adoption of knowledge sharing practices based on two fuzzy sets. The system uses the type of activity and the level of activity to codify the membership value of a user to a certain category. In previous work, users are described as undergoing a change process that brings them from their old practices to the conscious adoption of knowledge management practices (e.g. transition from low or non-existing levels of knowledge sharing practices to the widespread adoption of best behaviors in knowledge sharing) through different types of agent-based interventions [55].

The membership of a candidate $\mathrm{x}$ in a category is calculated as a function $\mathrm{Y}=\mathrm{f}\left(\mathrm{x}_{1}, \mathrm{x}_{2}\right)$, where:

$\mathrm{Y}$ - the level of knowledge sharing is fuzzified as:[very high, high, medium, low, very low] These values can be mapped into five categories: [adopter, trial, interested, aware, unaware].

$\mathrm{x}_{1}$ - the type of activity, is fuzzified as: [high, medium, low] which can be mapped into the stereotypes defined above [writer, reader, lurker]. 
$\mathrm{x}_{2}$ - the level of activity, is fuzzified as: [high, medium, low, very low] which corresponds to the categories [very active, active, visitor, inactive]

The columns of table 1 correspond to the linguistic variables for the level of activity and the rows correspond to the linguistic variables assigned to the type of activity. The rules are defined similar to a fuzzy controller. No valid rules are applied to the grey cells.

Table -1. The calculus of the level of knowledge sharing

\begin{tabular}{|l|l|l|l|l|}
\hline $\mathrm{Y}=\mathrm{f}\left(\mathrm{x}_{1}, \mathrm{x}_{2}\right)$ & high & medium & low & very low \\
\hline high & $\begin{array}{l}\text { very } \\
\text { high }\end{array}$ & very high & medium & \\
\hline medium & high & medium & low & \\
\hline low & & & very low & very low \\
\hline
\end{tabular}

Translated from the table, the classifier system uses the following rules:

If $x_{1}$ is high and $x_{2}$ is high then $Y$ is very high If $x_{1}$ is high and $x_{2}$ is medium then $Y$ is very high

If $x_{1}$ is high and $x_{2}$ is low then $Y$ is medium

If $x_{1}$ is medium and $x_{2}$ is high then $Y$ is high

If $\mathrm{x}_{1}$ is medium and $\mathrm{x}_{2}$ is medium then $\mathrm{Y}$ is medium

If $\mathrm{x}_{1}$ is medium and $\mathrm{x}_{2}$ is low then $\mathrm{Y}$ is low

If $x_{1}$ is low and $x_{2}$ is very low then $Y$ is very low

By defuzzifing the rules above, users are classified according to the level of knowledge sharing into five categories. As introduced above, using Near's terminology and mapping it into Rogers' theory related to the diffusion of innovation [54] the following user states related to the level of adoption of knowledge sharing behaviors can be identified: unaware, aware, interested, trial and adopter.

\subsection{Exploiting Metadata and User Modeling}

Data and metadata can be used for different scenarios including: personalization, learning and change management, networking and computer supported collaborative work, expertise discovery [56]. In the following we present an example of how metadata and user modeling can be exploited for the management of competencies and management of the tacit knowledge. It has been shown previously that the user ontology describes various properties and concepts relevant for the user model. The concepts of the user ontology are bridged with the concepts of the domain ontology through properties. Figure 4 depicts a part of the user ontology in a graph-based representation. Properties of concepts, such as "works_at", "works_on", "cooperates_with", associated with ontology specific reasoning mechanisms, facilitate further inferences. For instance, the fact that: "Smith works_on Ontologging project". Given the fact that the range of the property "works_on" is restricted to the concept Project and Ontologging is described as a project about: Knowledge Management, User Modeling and Semantic Web. Based on these facts an ontology-based system can automatically infer that Smith might be interested in or has expertise in: Semantic Web, Knowledge Management and User Modeling.

(User, works_on, Project)
(Project, related_to, Topic)

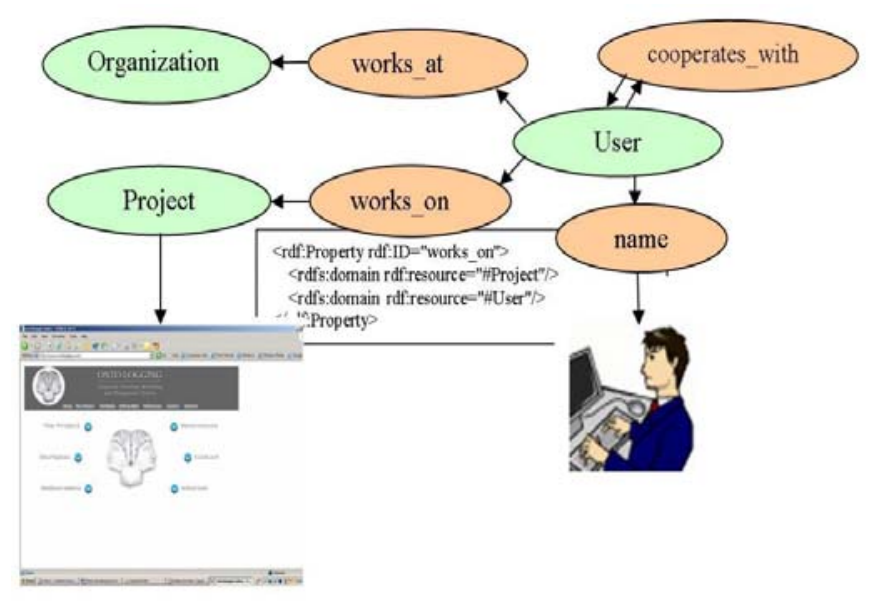

Figure 4 Application scenario of the user ontology

In our examples the previous RDFS tuples are instantiated as following:

(Smith, works_on, Ontologging)

(Ontologging, related_to, Knowledge Management)

(Ontologging, related_to, Ontology)

(Ontologging, related_to, User modeling)

Using associated reasoning mechanisms, an ontology-based KMS can deduce that Smith might be an expert in Knowledge Management, Ontology and User modeling. Thus, without requiring people to constantly update their profiles (their expertise, interests), an ontology-based KMS could facilitate finding the experts, knowledgeable persons in a domain or domains of interests for the users. Such mechanisms would enable to make explicit some of the competencies that a user might not be aware of and might help knowledge workers, better manage their competencies and skills and thus integrate personal knowledge management features into a KM platform. Furthermore the inferred user's expertise and interests can be used for pushing relevant knowledge, creating communities of practice or learning networks where experts and peers can collaborate, interact, communicate or share knowledge. The overall architecture of a semantic-enhanced knowledge management platform emphasizing the points of entry for the user modeling system has been presented in previous publications [38].

\section{Discussion AND EVAluation RESUlts}

In the recent years, semantic-enhanced user models are built following an ad-hoc approach, bottom-up or top-down methodology. Many user ontologies follow an ad-hoc approach in their development, taking into account specific goals, requirements of specific applications domains and don't present details on how the ontology was built [43]. Ontologies may be constructed based on the user's browsing behavior, they may consist of user interests inferred concepts, as in the 
context of web search [57]. Another bottom-up approach in constructing ontologies is based on automatic metadata extraction from folksonomies, user-annotations [58] or tags [59]. For example, user-specific annotation of content may be used to infer user's interests and preferences and used for personalized recommendations of content [58]. Contrary to the bottom-up approach, the OntobUM user ontology follows a top-down approach based on the IMS LIP specification [60]. In the context of semantic web, ontologies are meant to be extended, reused and integrated from different sources. The advantage of using a specification-based approach in developing an ontology is that it complies with a general model, an agreed conceptualization and therefore it is interoperable in the sense that it encompasses a set of general concepts that may be exchanged between different applications, extended or enriched to accommodate different types of applications. A similar, specification-based approach is proposed by [16]. This learner ontology proposed in Elena project is developed for e-learning applications, using IEEE PAPI [61] learner profile standard. Associated with the learner ontology, a framework for browsing, manipulating and maintaining interoperable learner profiles has been proposed for e-learning applications in [16]. More recently, a personal ontology for personal information management systems has been proposed in [34]. The authors point out that creating a personal ontology automatically, manually or semiautomatically is not an easy task. Furthermore, a personal ontology needs to reflect the user individually while it should comply with a general model that enable to exchange information between users and at the same time be usable by computer. An overview of various existing semantic-driven systems taking into account adaptive hypermedia tasks and sub-tasks and semantic web technologies is proposed in [62]. Comparing different user models is not an easy task because user models may be presented at a high level, so user models features might not be presented in details or in certain cases may not be comparable [47]. According to the best of our knowledge, and as presented in the above mentioned survey, no model of user behavior in the context of KM has been presented so far. Previous related work focuses either on skill management [29], or on users' information needs and the associated tasks and roles [28]. Furthermore, as presented in section 2 and 3.1, no work proposes a framework for modeling user behavior in the context of KM. As already pointed out in the introduction of the article, user modeling may contribute to bridge the gap between $\mathrm{KM}$ and personal $\mathrm{KM}$ in order to better support knowledge work and associated learning processes.

The identification of the objectives, features the system is expected to support and its associated research questions are elements that contribute to the selection of the evaluation methodology. In the context of a KM, business knowledge processes used in organizations such as knowledge creation, knowledge acquisition, knowledge structuring, knowledge capitalization, knowledge searching, knowledge sharing and the support of social/collaborative processes, are important factors to be considered. These particular aspects are to be associated with the short-term and/or long-term objectives a specific organization follows, they can provide insights for the design process itself (summative evaluation) for the functioning system (formative evaluation) or for its effectiveness defined also as substantive value of the solution. The overall evaluation of the Ontologging system focused on the efficiency and effectiveness of an ontology-based KMS. The evaluation of the Ontologging system has mainly taken place at two Spanish companies, a big multinational Information Technology consultancy company and a smaller IT company specialized in the design and implementation of enterprise software products (such as accounting, people management, knowledge management software). The profiles of the employees participating in the experiments were diverse (consultants, software analysts, system architects), and the employees originate from different domains (security, ERP, etc.). The evaluation of the system has been done combining the usage data analysis with questionnaires and other empirical evaluation methods including user studies and semistructured interviews. Taking into account the objectives of the article we will limit the scope of the analysis of the evaluation results and we will further discuss some results of the evaluation pertaining to the specific objectives of this article. A more complete description of the whole evaluation process, including the methodology, questionnaires and evaluation results can be found in the Ontologging system evaluation report [63]. The evaluation was limited for several reasons, among which also the fact that most of the documents were still in the old KMS and the transfer of data to a new system is not a straightforward process and the availability of the knowledge workers was limited. As pointed out in [48] statistical tools or models are often used when the quantity of data increases, even if semantic description is the main goal of the study. The focus of the evaluation towards was the effectiveness of the approach (what is the main value delivered to the users?), rather than the efficiency of the technical infrastructure (are the tools functioning well?) that has been developed. As a consequence, it was decided to use a more qualitative form of evaluation. Data has been gathered through several focus group discussions, semi-structured interviews, telephone interviews and several questionnaires. A number of questionnaires have been elaborated to collect the data: a pre-questionnaire (in Spanish) aimed to make a very early assessment of the situation and of the users' needs; the pre-questionnaire was designed and distributed to obtain a good understanding of the users participating in the test; a questionnaire constructed to evaluate the Ontologging knowledge structuring and content population process; a questionnaire dedicated to evaluate the user-centered tools of the Ontologging system, including the user modeling framework and knowledge distribution agents by the final end-users. The questionnaires included both closed questions (the user has to select a choice) and open questions (the user is asked to use free text to answer). An example of an answer indicating the definition of knowledge tools is given below: "Google may not be KM in the pure sense as I understand it, but it definitely solves most of my problems most of the time." The pre-questionnaire has been used to collect data related to the end-users' knowledge and practices of knowledge management. This questionnaire was distributed to the group of users before the system was deployed, and 14 
questionnaires have been collected. Extracts of the answers provided to the questionnaire related to the $\mathrm{KM}$ definitions and suggested associated problems are given bellow:

"For me a KM tool is the one that allows me to access the information I need to perform the tasks associated with my job in a fast and efficient manner".

"KM should help people benefit from experience not to have to redo things again".

"Part of the problem is to have a system in which people are willing to contribute."

KM tools don't improve work. "That's because currently it's necessary to spend too much time looking for material."

The answers to the pre-test questionnaires revealed the perceived needs and expectations of the end-users. Users expect KMS to help them easily access information, reuse past experience, save time, and locate experts. The following paragraph quotes some answers of the pre-questionnaire organized according to the most important issues that have been identified as the main pain points for the knowledge workers:

- To have timely access to information

"To facilitate access to information/knowledge relevant to the current work tasks in order to optimize work processes and improve productivity";

"The most important function is to facilitate access (on time) to [access] useful information in order to make my work easier and best."

"Searching the information that I need" "Discover and consume the knowledge generated by the company that could help me in my everyday tasks."

"To get briefings of news"

- To better re-use past experience

"Re-use past experience"

"Reuse company's past experience: documents, experiences, etc."

"Not losing time studying problems which have been solved before."

- To save time

"Saving time when I am searching for a solution."

"When I must do a tender, I need a lot and diverse information about products, prices, references, news, etc. There is, normally, little time to do it in and to have information on time if it is necessary."

"A major advantage is saving time when I'm searching for a solution."

"The most important functionality of this system is that it saves me time in searching any kind of information that I need."

- To find experts and collaborate

"Look for experts in order to ask for tacit knowledge."

"Find the right people to solve concrete problems."

"Also some KM tool should provide information to know what people know about something or has a previous experience with some technologies and products".

"Direct chat with experts".

In conclusion, as discussed in the previous section, user modeling in KM relates to a number of important issues such as the information overload issue, expressed as the need for enhanced user support, personalization, collaboration support, the need to better manage the tacit knowledge and the need to find experts. The need for enhanced user support for filtering and retrieving the knowledge available in the system expressed as "to not get lost" amongst hundreds of documents and to filter "information and noise" underlies the need of better knowledge management tools that support personal knowledge management and personalization. The main issues addressed by the evaluation of the advanced user centered usages were: employees' view on user modeling processes, the perceived need of personalization and associated incentives for knowledge sharing.

End-user's view on user modeling

Personalized systems require users to submit user data (personal information). The disclosure of user data opens up a series of problems like privacy and security but it also opens up new forms of personalization, communication, collaboration and social interactions. In our case the user profile editor enables the users to enter and update personal information and thus instantiate the user ontology. The user is in control of his/her "user profile" data. The user profile editor also enables users to visualize another person's profile in order to support collaboration and communication between the employees. User modeling techniques enable to capture certain characteristics of the users mapped under the Behavior concept. The evaluation of the system has emphasized that the integration of the user models and user modeling in KMSs is a sensitive issue. The questionnaires and semi-structured interviews with the end-user underlined the fact that certain users are concerned with privacy and trust issues. This category of users seems to be reluctant to allow the organization to use their data. Therefore according to the user opinion the user profiles should be made partially available to the other end-users and fully available to human resources.

Motivation for knowledge sharing

The behavior of the users can be associated with incentives to share, create knowledge or be active in the system. Of course the issue of sharing knowledge, creating knowledge or contributing to the system is complex and shouldn't be limited to simple incentives (e.g., reputation, ranking, visibility, promotion, bonus). It might imply changes of the current work practices and it can be associated with other managerial interventions. We have surveyed different types of incentives a company might use to stimulate knowledge sharing and knowledge creation. According to the user's opinion, reputation and promotion in organization would be the right incentives to stimulate a knowledge sharing culture in the organization. However a bonus associated with the salary also seems to be a good incentive for experts to spend extra time sharing their knowledge. Some expert knowledge workers have expressed their concern in being recognized as experts and having to do extra work.

A totally new vision has emerged related to the perception of the usage of a semantic-enhanced knowledge management system than what was originally envisaged. Ontology-based modeling and semantic web technology enables to go beyond traditional knowledge management system. It is possible to support more complex knowledge-oriented processes by 
exploiting the metadata and the relationships between the concepts (concept-based navigation). The advantage is the power of the relationships which enables users to navigate easily from one concept and its instances to another concept and its instances. Metadata and ontology-based representations connect knowledge resources with people. In the user ontology, people are modeled as authors of the documents or contributors, through relationships such as: "is_author", "has_contributor". People are connected with other people through "collaborates_with" and "works_with" type of relationships. These relationships constitute contextual links among the various chunks of content and enable to provide a certain support for the management of the tacit knowledge within organizations. The social and personal dimension will be important features for a next generation of KMS. A personal knowledge management will help knowledge workers manage their competencies, achieve their goals, fulfill work tasks and support social processes and collaboration with peers and experts or support change management processes. A user ontology, which could be named a semantic e-portfolio [64], could support such goals. The user ontology along with user modeling processes will support a more human-centric or user-centric approach of Semantic Web. Semantic Web can be foreseen to provide more relevant content for the users integrating different sources of information, using mash-ups or other personalized recommendations in order to better harness collective knowledge, to reduce information overload and support attention management, to better support users in searching for information and make recommendations of relevant content using collective intelligence, to better support lifelong learning and personal knowledge management, and/or to better help users to achieve their goal. Personalization will be one of the defining characteristics of a next generation of services where semantics of data will play a key role along with specific goals or characteristics of the users stored in a user ontology.

\section{CONCLUSIONS AND FUTURE WORK}

The usage and application of ontologies is increasingly seen as a key to enable semantics-driven data access and processing [36] and human semantic web vision. This article has presented an integrated framework for modeling users based on ontologies, OntobUMf. User modeling processes are targeted to support key knowledge processes and motivate people creating and sharing knowledge, to facilitate collaboration between knowledge workers irrespective of their location and better harness collective intelligence, to alleviate information overload, to simplify business processes and to better support work tasks and change management. The Behavior concept was introduced as an extension of IMS LIP in order to model the behavior of the user-the knowledge providers of the system. A model of the behavior of the user is proposed and the classification of the users based on the level of knowledge sharing has been described and implemented using the principles of a fuzzy classifier system. OntobUMf classify the users according to the level of activity, type of activity and level of knowledge sharing. The classification process takes into account the level of activity and the type of activity, characteristics of the users inferred on the basis of the interaction of the user with a knowledge management system. Based on the type of activity the users are classified into: readers, writers, lurkers. Based on the level of activity the users are classified as: very active, active, visitor, inactive. Based on the level of knowledge sharing users can be: unaware, aware, interested, trial or adopter. In previous work, users are described as undergoing a change process that brings them from their old practices to the conscious adoption of knowledge management practices (e.g. transition from low or non-existing levels of knowledge sharing practices to the widespread adoption of best behaviors in knowledge sharing) [55]. This framework is suitable to be adapted and extended in other application domains such as e-learning, competency management, human resource management or decision support. User modeling makes a personal KM possible and it could facilitate users a better control of their activities and would enable to provide them feedback that could be used to help users achieve their short term or long term goals. A system which can keep track of their actions, activities and knowledge processes could model their behavior and thus such a system will provide them with a better understanding of their individual actions, and/or could even play the role of a change agent in various types of platforms [55, 65], simulations, or games [66]. Nowadays a knowledge worker needs to be self-sufficient, sometimes needs to unlearn unnecessary knowledge and old habits that get in the way of working smarter and not harder [67].

Semantic-enriched resources and ontologies can support the development of a new range of services to enhance user support and lifelong learning. Integration of associated reasoning mechanisms can open up the possibility of making knowledge assets intelligently accessible and associate various types of intelligent, personalized services and create a next generation of services in a corporate setting or on the web.

\section{REFERENCES}

[1] C. Holsapple, W., "The inseparability of modern knowledge management and computer-based technology," Journal of Knowledge Management, vol. 9, pp. 42-52, 2005.

[2] M. Alavi and D. E. Leidner, "Review: Knowledge Management And Knowledge Management Systems: Conceptual Foundations And Research Issues," MIS Quarterly, vol. 25, pp. 107-136, 2001.

[3] J. Damsgaard and R. Scheepers, "Harnessing Intranet Technology for Organizational Knowledge creation," Australian Journal of Information Systems, pp. 4-15, 2001.

[4] E. Truch, "Managing personal knowledge: The key to tomorrow's employability," Journal of Change Management, vol. 2, pp. 102105, 2001.

[5] A. Garcia-Crespo, R. Colomo-Palacios, J. M. Gomez-Berbis, and B. Ruiz-Mezcua, "SEMO: a framework for customer social networks analysis based on semantics," J Inf technol, vol. 25, pp. 178-188, 2010.

[6] S. Schaffert, "IkeWiki: A semantic wiki for collaborative knowledge management," in International Workshop on Semantic 
Technologies in Collaborative Applications (STICA'06), Manchester, UK, 2006, pp. 388-396.

[7] E. Bertino, L. R. Khan, R. Sandhu, and B. Thuraisingham, "Secure knowledge management: confidentiality, trust, and privacy," Systems, Man and Cybernetics, Part A: Systems and Humans, IEEE Transactions on, vol. 36, pp. 429-438, 2006.

L. Razmerita, K. Kirchner, and F. Sudzina, "Personal Knowledge Management: The Role of Web 2.0 tools for managing knowledge at individual and organisational levels," Online Information Review, vol. 33, pp. 1021-1039, 2009. Collective Knowledge Management in the Web 2.0: Two Faces of Knowledge Management," in 9th International Conference on Innovative Internet Community Systems, Jena, Germany, 2009, pp. $15-26$.

[10] L. Razmerita, "User Modeling and Personalization of Knowledge Management Systems," in Adaptable and Adaptive Hypermedia, S. Y. Chen and G. D. Magoulas, Eds.: Idea Group Publishing, 2005, pp. 225-245.

[11] A. Oulasvirta and J. Blom, "Motivations in personalisation behaviour," Interacting with Computers, vol. 20, pp. 1-16, 2008.

[12] A. Maedche, B. Motik, and L. Stojanovic, "Managing multiple and distributed ontologies on the Semantic Web," VLDB Journal, vol. 12, pp. 286-302, 2003.

[13] D. Allemang and J. A. Hendler, Semantic web for the working ontologist: Morgan Kaufmann, 2008.

[14] B. Motik, I. Horrocks, R. Rosati, and U. Sattler, "Can OWL and Logic Programming Live Together Happily Ever After? ," in Proc. of the 5th Int. Semantic Web Conference (ISWC 2006). vol. 4273, I. Cruz, F., S. Decker, D. Allemang, C. Preist, D. Schwabe, P. Mika, M. Uschold, and A. Lora, Eds. Athens, USA: LNCS, Springer, 2006, pp. 501-514.

[15] A. Naeve, "The Human Semantic Web Shifting from Knowledge Push to Knowledge Pull," Int. Jornal of Semantic Web Inf. Syst., vol. 1, pp. 1-30 2005 .

[16] P. Dolog and M. Schäfer, "A framework for browsing, manipulating and maintaining interoperable learner profiles," in Proc. User Modeling 2005: 10th International Conference, UM 2005, 2003, pp. 397-401.

[17] D. Heckmann, Schwartz, T., Brandherm, B., Schmitz, M., and von Wilamowitz-Moellendorff, M., "GUMO - the General User Model Ontology," in 10th International Conference on User Modeling (UM'2005), Edinburgh, UK, , 2005, pp. 428-432.

[18] D. Heckmann, E. Schwarzkopf, J. Mori, D. Dengler, and A. Kroner, "The User Model and Context Ontology GUMO revisited for future Web 2.0 Extensions." vol. Contexts and Ontologies: Representation and Reasoning, 2007, pp. 37-46.

[19] M. Hatala, R. Wakkary, and L. Kalantari, "Rules and ontologies in support of real-time ubiquitous application," Web Semantics: Science, Services and Agents on the World Wide Web, vol. 3, pp. 522, 2005.

[20] S. Gauch, J. Chaffee, and A. Pretschner, "Ontology-based personalized search and browsing," Web Intelligence and Agent Systems, vol. 1, pp. 219-234, 2003.

[21] S. Gauch, M. Speretta, A. Chandramouli, and A. Micarelli, "User Profiles for Personalized Information Access," in The Adaptive Web, Methods and Strategies of Web Personalization, P. Brusilovsky, A. Kobsa, and W. Nejdl, Eds.: Springer, 2007, pp. 5489.

[22] L. Razmerita and M. Lytras, "Ontology-Based User Modelling Personalization: Analyzing the Requirements of a Semantic Learning Portal," in Emerging Technologies and Information Systems for the Knowledge Society. vol. 5288 2008: Springer Berlin/Heidelberg, 2008, pp. 354-363.
[23] N. Henze, P. Dolog, and W. Nejdl, "Reasoning and Ontologies for Personalized E-Learning in the Semantic Web," Educational Technology \& Society, vol. 7, pp. 82-97, 2004.

[24] P. Dolog and W. Nejdl, "Semantic Web Technologies for the Adaptive Web," in The Adaptive Web, P. Brusilovsky, A. Kobsa, and W. Nejdl, Eds.: Springer, 2007, pp. 697-719.

[25] M. A. Sicilia, M. D. Lytras, E. Rodríguez, and E. GarcíaBarriocanal, "Integrating descriptions of knowledge management learning activities into large ontological structures: A case study," Data \& Knowledge Engineering, vol. 57, pp. 111-121, 2006.

[26] M. Vargas-Vera and M. Lytras, D.,, "Exploiting semantic web and ontologies for personalised learning services: towards semantic web-enabled learning portals for real learning experiences," International Journal Knowledge and Learning, vol. 4, pp. 1-17, 2008.

M. A. Sicilia and M. D. Lytras, "The semantic learning organization," The Learning Organization, Emerald vol. 12, pp. 402-410, 2005.

[28] L. Van Elst, A. Abecker, and H. Maus, "Exploiting user and process context for knowledge management systems," in Workshop on User Modeling for Context-Aware Applications, the 8th Int. Conf. on User Modeling, Sonthofen, Germany, 2001.

[29] Y. Sure, H. Akkermans, J. Broekstra, J. Davies, Y. Ding, A. Duke, R. Engels, D. Fensel, I. Horrocks, V. Iosif, A. Kampman, M. Kiryakov, M. Klein, T. Lau, D. Ognyanov, U. Reimer, K. Simov, R. Studer, J. van der Meer, and F. van Harmelen, "On-ToKnowledge: Semantic Web Enabled Knowledge Management," in Web Intelligence, 2008, pp. 277-300.

[30] C. W. Chan, "FROM KNOWLEDGE MODELING TO ONTOLOGY CONSTRUCTION," International Journal of Software Engineering \& Knowledge Engineering, vol. 14, pp. 603624, 2004.

[31] L. Jaehyun, S. Hyowon, and H. Soon Hung, "Ontology-based Knowledge Framework for Product Development," ComputerAided Design \& Applications, vol. 2, pp. 635-643, 2005.

[32] G. A. Pisanelli D.M., Steve G., "Ontologies and Information Systems: the Marriage of the Century?," in Proceedings of Lyee Workshop Paris, 2002.

[33] L. Stojanovic, A. Maedche, B. Motik, and N. Stojanovic, "UserDriven Ontology Evolution Management," in EKAW-2002. vol. Proceedings of the 13th European Conference on Knowledge Engineering and Management, EKAW-2002, Springer, LNAI, Madrid, Spain, 2002.

[34] A. Katifori, C. Vassilakis, I. Daradimos, G. Lepouras, Y. Ioannidis, A. Dix, A. Poggi, and T. Catarci, "Personal Ontology Creation and Visualization for a Personal Interaction Management System," in CHI 2008 Workshop Personal Information Management PIM2008, Florence, 2008.

[35] H. H. Kim, S. Y. Rieh, T. K. Ahn, and W. K. Chang, "Implementing an ontology-based knowledge management system in the Korean financial firm environment," in Proceedings of the 67th Annual Meeting of the American Society for Information Science and Technology. , Medford, N.J., USA, 2004, pp. 300309.

[36] A. Maedche, B. Motik, L. Stojanovic, R. Studer, and R. Volz, "Ontologies for Enterprise Knowledge Management " IEEE Intelligent Systems, vol. 18, pp. 26-33, 2003.

[37] V. Uren, P. Cimiano, J. Iria, S. Handschuh, M. Vargas-Vera, E. Motta, and F. Ciravegna, "Semantic annotation for knowledge management: Requirements and a survey of the state of the art," Web Semantics: Science, Services and Agents on the World Wide Web, vol. 4, pp. 14-28, 2006.

[38] L. Razmerita, "Ontology-based User Modeling for Knowledge Management Systems," in Ontologies: A Handbook of Principles, 
Concepts and Applications in Information Systems, R. Sharman, R. Kishore, and R. Ramesh, Eds.: Springer, 2007, pp. 635-664.

[39] L. Razmerita, A. Angehrn, and A. Maedche, "Ontology-based user modeling for knowledge management systems," in User Modeling 2003, Proceedings. vol. 2702, 2003, pp. 213-217.

[40] A. Kobsa, Koenemann, J., and Pohl, W.,, "Personalized hypermedia presentation techniques for improving online customer relationships," The Knowledge Engineering Review vol. 16, pp. 111-155, 2000.

[41] S. Gerogiannakis, M. Sintichakis, and N. Achilleopoulos, "Collaborative Knowledge Management and Ontologies The ONTO-LOGGING Platform," in Knowledge Management in Electronic Government, 2003, pp. 616-616.

[42] G. Boella and L. Van Der Torre, "Security policies for sharing knowledge in virtual communities," Systems, Man and Cybernetics, Part A: Systems and Humans, IEEE Transactions on, vol. 36, pp. 439-450, 2006.

[43] L. Razmerita, R. Firantas, and M. Jusevičius, "Towards a New Generation of Social Networks: Merging Social Web with Semantic Web," in 5th International Conference on Semantic Systems, Graz, Austria, 2009, pp. 412-423.

[44] M. Uschold and M. Gruninger, "Ontologies: principles, methods, and applications," Knowledge Engineering Review, vol. 11, pp. 93$155,1996$.

[45] IMS Consortium, "IMS Learner Information Package Specification," 2003.

[46] L. Razmerita, "Modeling Behavior of Users in Semantic-enhanced Information Systems: The role of a User Ontology," in Authoring of Adaptive and Adaptable Hypermedia Workshop, in Adaptive Hypermedia Hannover, 2008, pp. 69-77.

[47] A. Brun, A. Boyer, and L. Razmerita, "Compass to Locate the User Model I need: Building the Bridge between Researchers and Practitioners in User Modeling," in 18th International Conference, User Modeling and User Adaptive Interaction, UMUAI 2010, P. De Bra, A. Kobsa, and C. D., Eds. Hawaii, USA: LNCS, Springer, 2010, pp. 303-314.

[48] L. Lancieri and N. Durand, "Internet user behavior: compared study of the access traces and application to the discovery of communities," Systems, Man and Cybernetics, Part A: Systems and Humans, IEEE Transactions on, vol. 36, pp. 208-219, 2005.

[49] E. Brown, T. Brailsford, T. Fisher, and C. v. d. Eijk, "Revealing the hidden rationality of user browsing behaviour," in Proceedings of the eighteenth conference on Hypertext and hypermedia Manchester, UK: ACM, 2007, pp. 85-94.

[50] M. Graff, "Individual differences in hypertext browsing strategies," Behaviour \& Information Technology, vol. 24:2, pp. 93-99, 2005.

[51] L. Clark, I. Ting, C. Kimble, P. Wright, and D. Kudenko, "Combining ethnographic and clickstream data to identify user Web browsing strategies," Information Research, vol. 11:2, 2006.

[52] P. Kollock, "Social dilemmas: The anatomy of cooperation," Annual Reviews in Sociology, vol. 24, pp. 183-214, 1998.

[53] A. Cabrera and E. F. Cabrera, "Knowledge-sharing dilemmas," Organization studies, vol. 23, pp. 687-710, 2002.

[54] E. Rogers, "Diffusion of Innovation," NY, 1962.

[55] C. Roda, A. Angehrn, T. Nabeth, and L. Razmerita, "Using conversational agents to support the adoption of knowledge sharing practices," Interacting with Computers, Elsevier, vol. 15, pp. 57-89, 2003.

[56] L. Razmerita, "Exploiting Semantics and User Modeling for Enhanced User Support, ," in HCI International (Human Computer Interaction) Conference. vol. Proceedings of HCI International 2005 Las Vegas, USA, 2005.
S. Gauch, J. Chaffee, and A. Pretschner, "Ontology-Based User Profiles for Search and Browsing," User Modeling and UserAdapted Interaction: The Journal of Personalization Research, Special Issue on User Modeling for Web and Hypermedia Information Retrieval, 2003.

[58] A. Nauerz, F. Bakalov, B. Konig-Ries, and M. Welsch, "Personalized Recommendation of Related Content Based on Automatic Metadata Extraction," in Proceedings of the 2008 conference of the center for advanced studies on collaborative research: meeting of minds, Ontario, Canada, 2008.

[59] F. Carmagnola, F. Cena, O. Cortassa, C. Gena, and I. Torre, "Towards a Tag-Based User Model: How Can User Model Benefit from Tags?," in Proceedings of the 11th international conference on User Modeling Corfu, Greece: Springer-Verlag, 2007.

[60] IMS Consortium, "IMS LIP, Information Management Systems Learner Information Packaging Information Model Specification, last retrieved in April 2008 from: http://www.imsglobal.org/profiles/lipinfo01.html," 2001.

[61] IEEE Consortium, "IEEE PAPI, Draft Standard for Learning Technology - Public and Private Information (PAPI) for Learners (PAPI Learner)," 2000.

[62] I. Torre, "Adaptive systems in the era of the semantic and social web, a survey," User Modeling and User Adapted Interaction, vol. 19, pp. 433-486, 2009.

[63] T. Nabeth and L. Razmerita, "Final Evaluation report of the use of Onto-Logging platform in the user site 1.0; Ontologging project deliverable," CALT, INSEAD 2004.

[64] L. Razmerita, G. Gouarderes, and E. Comte, "Ontology-based User Modeling and e-Portfolio Grid Learning Services," Applied Artificial Intelligence Journal, vol. 19, pp. 905-931, 2005.

[65] A. Angehrn, Nabeth, T., Razmerita, L., Roda, C., , "K-InCA: Using Artificial Agents for Helping People to Learn New Behaviours," vol. Proc. of IEEE International Conference on Advanced Learning Technologies (ICALT 2001), 2001.

[66] A. A. Angehrn, "Learning to manage innovation and change through organizational and people dynamics simulations," in Proceedings of the International Simulation \& Gaming Association Conference (ISAGA05), Atlanta, USA, 2005.

[67] M. Cope, "Know Your Value?," P. Education, Ed. London: Prentice Hall, 2000.

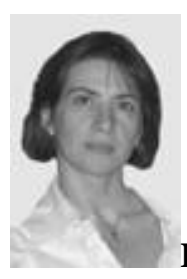

Liana Razmerita is Assistant Professor at Copenhagen Business School in Denmark. She has received her B.S. and M.S. from the "Dunarea de Jos" University, Galati, Romania. She holds a PhD degree in computer science from Paul Sabatier University, Toulouse, France. Her doctoral research work was done on the topic of Ontology-based User Modeling for Knowledge Management Systems at the Centre of Advanced Learning Technologies, INSEAD, France. Previous publications include more than 40 peer-reviewed journal articles, book chapters and conference papers in domains such as: user modeling, social/semantic web, e-learning, knowledge management and e-government. 